\title{
On the cosmological constant: its identification as renormalization group invariant scale corresponding to a gravitational condensate
}

\author{
Herbert W. Hamber \\ Department of Physics and Astronomy \\ University of California, Irvine \\ Irvine, California 92697-4575, USA \\ E-mail: HHambereuci.edu \\ Reiko Toriumi* \\ Centre de Physique Théorique UMR 7332 \\ Campus de Luminy, Case 907, 13288, Marseille, France \\ E-mail: Reiko. Toriumi@cpt.univ-mrs.fr
}

\begin{abstract}
We examine the general issue of whether a scale dependent cosmological constant can be consistent with general covariance, a problem that arises naturally in the treatment of quantum gravitation where coupling constants generally run as a consequence of renormalization group effects. The issue is approached from several points of view, which include the manifestly covariant functional integral formulation, covariant continuum perturbation theory about two dimensions, and the lattice formulation of gravity. In all cases we find that the cosmological constant cannot run with scale, unless general covariance is explicitly broken by the regularization procedure. This article is based on Int. J. Mod. Phys. D22 (2013) 13, 1330023 [arXiv:1301.6259].
\end{abstract}

Frontiers of Fundamental Physics 14 - FFP14,

15-18 July 2014

Aix Marseille University (AMU) Saint-Charles Campus, Marseille

\footnotetext{
* Speaker.
} 


\section{Introduction}

We present the properties of the cosmological constant suggested by the quantum field theory methods, in particular we focus on the Wilson's renormalization group studies to analyze the role of the cosmological constant in the framework of pure Einstein gravity with the cosmological constant term. We will first analyze how the bare cosmological constant which appears in the original Lagrangian should be absorbed into the physical coupling constant by rescaling the gravitational field. We then argue that the dynamically generated RG invariant scale should be identified with the observed cosmological constant. This scale can be interpreted as a gravitational condensate. Specifically, the plan to achieve the above discussion is as follows: we first discuss how the proper rescaling of the metric is equivalent to removing the gauge dependence of the renormalized coupling constants which results in only one physical coupling constant. We discuss analogies with relevant aspects from non-Abelian (Yang-Mills) gauge theory, QCD. The gravitational Wilson loop will be discussed, which supplements the indication of the role of the observed cosmological constant.

\section{Gauge Dependence in the Renormalization of the Cosmological Constant}

Perturbation theory generally serves a very useful purpose, as it allows one to systematically track the gauge dependence of various renormalization effects. Unfortunately Einstein gravity is not perturbatively renormalizable in four dimensions, so this easy route is not available. Nevertheless, in lower dimensions it is possible to rescue in part the perturbative treatment and partly address some of the key issues. One does not, of course, expect the answers to be quantitatively correct, however it becomes clear below that the issue of gauge invariance comes up, and is eventually successfully resolved. Let us emphasize here that one key aspect of the perturbative treatment via the background field method is that diffeomorphism invariance is carefully preserved throughout the calculation.

In two dimensions the gravitational coupling $G \sim \Lambda^{2-d}$ is dimensionless, and the theory appears to be perturbatively renormalizable. Despite the fact that the gravitational action reduces to a topological invariant in two dimensions, it is meaningful to try to construct, in analogy to Wilson's original suggestion for scalar field theories, the theory perturbatively as a double series in $\varepsilon=d-2$ and $G[1,4,5]$. The $2+\varepsilon$ expansion for pure gravity proceeds as follows [6]. First the gravitational part of the Lagrangian

$$
\mathscr{L}=-\frac{\Lambda^{\varepsilon}}{16 \pi G} \sqrt{g} R,
$$

with $G$ dimensionless and $\Lambda$ a microscopic ultraviolet cutoff, is expanded in the fields by setting $g_{\mu \nu} \rightarrow \bar{g}_{\mu \nu}=g_{\mu \nu}+h_{\mu \nu}$, where $g_{\mu \nu}$ is the classical background field and $h_{\mu \nu}$ the small quantum fluctuation. To make perturbation theory convergent requires a gauge fixing term, for example, in the form of a harmonic gauge condition, $\mathscr{L}_{g f}=\frac{1}{2} \alpha \sqrt{g} g_{v \rho}\left(\nabla_{\mu} h^{\mu v}-\frac{1}{2} \beta g^{\mu v} \nabla_{\mu} h\right)\left(\nabla_{\lambda} h^{\lambda \rho}-\right.$ $\left.\frac{1}{2} \beta g^{\lambda \rho} \nabla_{\lambda} h\right)$, with $h^{\mu v}=g^{\mu \alpha} g^{v \beta} h_{\alpha \beta}, h=g^{\mu v} h_{\mu \nu}$ and $\nabla_{\mu}$ the covariant derivative with respect to the background metric $g_{\mu \nu}$. In return, the gauge fixing term requires the introduction of a FaddeevPopov ghost contribution $\mathscr{L}_{\text {ghost }}$, giving the total Lagrangian to be $\mathscr{L}+\mathscr{L}_{\text {gf }}+\mathscr{L}_{\text {ghost }}$. Here we leave the two gauge parameters unspecified, so that later the detailed gauge dependence of the 
result can be checked. For the one loop divergences associated with the $\sqrt{g}$ and the $\sqrt{g} R$ terms, they obtain respectively

$$
\lambda_{0} \rightarrow \lambda_{0}\left[1-\left(\frac{a_{1}}{\varepsilon}+\frac{a_{2}}{\varepsilon^{2}}\right) G\right] \text { and } \frac{\mu^{\varepsilon}}{16 \pi \mathrm{G}} \rightarrow \frac{\mu^{\varepsilon}}{16 \pi \mathrm{G}}\left(1-\frac{\mathrm{b}}{\varepsilon} \mathrm{G}\right),
$$

with coefficients that depend on the gauge dependent parameters $\alpha$ and $\beta$, with $a_{1}=-\frac{8}{\alpha}+$ $8 \frac{(\beta-1)^{2}}{(\beta-2)^{2}}+4 \frac{(\beta-1)(\beta-3)}{\alpha(\beta-2)^{2}}, a_{2}=8 \frac{(\beta-1)^{2}}{(\beta-2)^{2}}$, and $b=\frac{2}{3} \cdot 19+\frac{4(\beta-1)^{2}}{(\beta-2)^{2}}[4,5]$. The gauge dependent artifact reflected in the renormalized couplings shown above can be beautifully compensated if one makes use of the freedom to rescale the metric, which is equivalently given by the field redefinition

$$
\left[1-\left(\frac{a_{1}}{\varepsilon}+\frac{a_{2}}{\varepsilon^{2}}\right) G\right] \sqrt{g}=\sqrt{g^{\prime}} \leftrightarrow g_{\mu v}=\left[1-\left(\frac{a_{1}}{\varepsilon}+\frac{a_{2}}{\varepsilon^{2}}\right) G\right]^{-2 / d} g_{\mu \nu}^{\prime},
$$

which restores the original unit coefficient for the cosmological constant term. By this procedure the cosmological term is brought back into its standard form $\lambda_{0} \sqrt{g^{\prime}}$, and one obtains for the complete Lagrangian to first order in $G$

$$
\mathscr{L} \rightarrow-\frac{\mu^{\varepsilon}}{16 \pi G}\left[1-\frac{1}{\varepsilon}\left(\frac{2}{3} \cdot 19\right) G\right] \sqrt{g^{\prime}} R^{\prime}+\lambda_{0} \sqrt{g^{\prime}},
$$

where only terms singular in $\varepsilon$ have been retained. From this last result one can finally read off the renormalization of Newton's constant $\frac{1}{G} \rightarrow \frac{1}{G}\left[1-\frac{1}{\varepsilon}\left(\frac{2}{3} \cdot 19\right) G\right]$. Therefore the gauge dependence has, as it should be on physical grounds, entirely disappeared from the final answer, which is simply due to general covariance. We make the point here that the results of covariant perturbation theory are entirely consistent with the scaling argument; only the renormalization of $G$ has physical meaning. Let us dwell further on this aspect.

In the presence of an explicit renormalization scale $\mu$ the only Callan-Symanzik $\beta$-function for pure gravity is obtained by requiring the independence of the effective coupling $G$ from the original renormalization scale $\mu$; to one loop order,

$$
\mu \frac{\partial}{\partial \mu} G(\mu) \equiv \beta(G)=\varepsilon G-\beta_{0} G^{2}+\mathscr{O}\left(G^{3}, \varepsilon G^{2}\right),
$$

with $\beta_{0}=\frac{2}{3} \cdot 19$. Depending on whether one is on the right $\left(G>G_{c}\right)$ or on the left $\left(G<G_{c}\right)$ of the nontrivial UV fixed point at $G_{c}=\frac{\varepsilon}{\beta_{0}}+\mathscr{O}\left(\varepsilon^{2}\right)$, the coupling will either flow to increasingly larger values of $G$, or flow towards the Gaussian fixed point at $G=0$, respectively. In the following we refer to the two phases as the strong and weak coupling phase, respectively. The running of $G$ as a function of momentum scale $\mu$ in pure gravity is obtained from integrating (2.5), giving

$$
G(\mu) \simeq G_{c}\left[1 \pm c_{0}\left(m^{2} / \mu^{2}\right)^{\varepsilon / 2}+\ldots\right],
$$

with $c_{0}$ a positive constant, and $m=\xi^{-1}$ a mass scale that arises as an integration constant of the renormalization group equations. The $\mu^{2}$-dependent contribution is the quantum correction, which at least within a perturbative framework is assumed to be small. The choice of + or - sign is determined from whether one is to the left $(-)$, or to right (+) of $G_{c}$, in which case the effective $G(\mu)$ decreases or, respectively, increases as one flows away from the UV fixed point towards 
lower momentum. Physically the two solutions represent screening $\left(G<G_{c}\right)$ and antiscreening $\left(G>G_{c}\right)$. While in the above continuum perturbative calculation both phases, and therefore both signs, look acceptable, the Euclidean and Lorentzian lattice results rule out the weak coupling phase as pathological, in the sense that there the lattice collapses into a 2 dimensional degenerate object $[7,8,9,14]$. This picture is consistent with the intuitive analysis of the properties of gravity; in gravity, as you go further in distance from the bare mass, more and more mass is included, therefore the effective mass should be bigger than the bare mass. ${ }^{1}$

The $\mu^{2}$-dependent quantum correction in (2.6) involves a new physical, RG invariant scale $\xi=$ $1 / m$ which cannot be fixed perturbatively, whose size then determines the distance scale relevant for quantum effects. In terms of the bare coupling $G(\Lambda)$, it can be expressed as

$$
\xi^{-1}(G) \equiv m=\Lambda \cdot A_{m} e^{-\int^{G(\Lambda)} \frac{d G^{\prime}}{\beta\left(G^{\prime}\right)}},
$$

with $A_{m}$ a constant. Note the rather remarkable fact that one scale has disappeared $\left(\lambda_{0}\right)$, and a new one has appeared dynamically $(\xi)$. Conversely, $\xi^{-1}=m$ is an RG invariant and one has

$$
\Lambda \frac{d}{d \Lambda} m[\Lambda, G(\Lambda)]=\mu \frac{d}{d \mu} m[\mu, G(\mu)]=0 .
$$

The constant $A_{m}$ cannot be determined perturbatively; it needs to be computed by nonperturbative (e.g., lattice) methods, for example by evaluating invariant correlations at fixed geodesic distances; it is related to the constant $c_{0}$ in (2.6) by $c_{0}=1 /\left(A_{m}^{1 / v} G_{c}\right)$. In the vicinity of the UV fixed point at $G_{c}$, for which $\beta\left(G_{c}\right)=0$, one can write $\beta(G) \equiv \mu \frac{\partial}{\partial \mu} G(\mu) \sim \beta^{\prime}\left(G_{c}\right)\left(G-G_{c}\right)+\ldots$ which by integration gives $\xi^{-1}(G) \propto \Lambda\left|\left(G-G_{c}\right) / G_{c}\right|^{v}$, with correlation length exponent $v=-1 / \beta^{\prime}\left(G_{c}\right)$. Note that the magnitude of $\xi$ is not determined by the magnitude of $G$. Instead, it is determined by the distance of the bare $G$ from the UV fixed point value $G_{c}$, and as such it can be arbitrarily large.

To summarize the results so far, we showed that the path integral for pure quantum gravity depends only on one dimensionless combination of couplings, $G \sqrt{\lambda_{0}}$ in $d=4$, and that the bare $\lambda_{0}$ is entirely scaled out of the path integral, and out of the physics. It is also clear that the only renormalization that is gauge independent and physically meaningful is the one for Newton's constant $G$. Finally, we emphasized the fact that the very same, manifestly covariant, RG treatment clearly shows the appearance of a new dynamically generated scale $\xi[(2.7)]$.

\section{The Gauge Theory Analogy}

$Q E D$ and $Q C D$ provide two invaluable illustrative cases where the running of the gauge coupling with energy is not only theoretically well understood, but also verified experimentally. As in $Q E D$, in $Q C D$ radiative corrections are known to significantly alter the behavior of the static potential at short distances. Changes in the potential are best expressed in terms of the running strong coupling constant $\alpha_{S}(\mu)$, whose scale dependence is determined by the celebrated $\beta$ function of $S U(3) Q C D$ with $n_{f}$ fermion flavors

$$
\mu \frac{\partial \alpha_{S}}{\partial \mu}=2 \beta\left(\alpha_{S}\right)=-\frac{\beta_{0}}{2 \pi} \alpha_{S}^{2}-\frac{\beta_{1}}{4 \pi^{2}} \alpha_{S}^{3}-\frac{\beta_{2}}{64 \pi^{3}} \alpha_{S}^{4}-\ldots,
$$

\footnotetext{
${ }^{1}$ Whereas in QED, where the virtual electron-positron clouds make the vacuum into dialectric medium, in which as you go further from the bare charge, the effective charge becomes smaller as it gets screened.
} 
with coefficients $\beta_{0}=11-\frac{2}{3} n_{f}, \beta_{1}=51-\frac{19}{3} n_{f}$, and $\beta_{2}=2857-\frac{5033}{9} n_{f}+\frac{325}{27} n_{f}^{2}$. The solution of the RG equation (3.1) then gives for the running of $\alpha_{S}(\mu)$

$$
\alpha_{S}(\mu)=\frac{4 \pi}{\beta_{0} \ln \mu^{2} / \Lambda_{\overline{M S}}^{2}}\left[1-\frac{2 \beta_{1}}{\beta_{0}^{2}} \frac{\ln \left[\ln \mu^{2} / \Lambda_{\overline{M S}}^{2}\right]}{\ln \mu^{2} / \Lambda_{\overline{M S}}^{2}}+\ldots\right] .
$$

The nonperturbative scale $\Lambda_{\overline{M S}}$ appears as an integration constant of the RG equations, and is therefore by construction scale independent. Indeed, the physical value of $\Lambda_{\overline{M S}}$ cannot be fixed from perturbation theory alone, and needs to be determined from experiment, which gives $\Lambda_{\overline{M S}} \simeq$ $213 \mathrm{MeV}$. In principle, one can solve for $\Lambda_{\overline{M S}}$ in terms of the coupling at any scale, and in particular at the cutoff scale $\Lambda$, obtaining

$$
\Lambda_{\overline{M S}}=\Lambda e^{-\int_{S}(\Lambda) \frac{d \alpha_{S}^{\prime}}{2 \beta\left(\alpha_{S}^{\prime}\right)}}=\Lambda\left(\frac{\beta_{0} \alpha_{S}(\Lambda)}{4 \pi}\right)^{\beta_{1} / \beta_{0}^{2}} e^{-\frac{2 \pi}{\beta_{0} \alpha_{S}(\Lambda)}}\left[1+O\left(\alpha_{S}(\Lambda)\right)\right]
$$

Not all physical properties can be computed reliably in weak coupling perturbation theory. In nonAbelian gauge theories a confining potential is found at strong coupling by examining the behavior of the Wilson loop, defined for a large closed loop $C$ as $\langle W(C)\rangle=\left\langle\operatorname{tr} \mathscr{P} \exp \left\{i g \oint_{C} A_{\mu}(x) d x^{\mu}\right\}\right\rangle$, with $A_{\mu} \equiv t_{a} A_{\mu}^{a}$ and the $t_{a}$ 's the group generators of $S U(N)$ in the fundamental representation. In the pure gauge theory at strong coupling, the leading contribution to the Wilson loop can be shown to follow an area law for sufficiently large loops

$$
\langle W(C)\rangle \underset{A \rightarrow \infty}{\sim} \exp \left(-A(C) / \xi^{2}\right),
$$

where $A(C)$ is the minimal area spanned by the planar loop $C$ [10]. The quantity $\xi$ is the gauge field correlation length, ${ }^{2}$ and is essentially the same [up to a factor $\mathscr{O}(1)$ ] as the inverse of $\Lambda_{\overline{M S}}$ in (3.3)

$$
\Lambda_{\overline{M S}} \sim \xi .
$$

The point here is that non-Abelian gauge theories are known to contain a new, fundamental, dynamically generated length scale, in clear analogy to the result of (2.7) for gravity. It is also understood that the inverse of the correlation length $\xi$ corresponds to the lowest gauge invariant mass excitation in the gauge theory, the scalar glueball (gauge field (gluon in QCD) condensate) with mass $m_{0}=1 / \xi$. As in the case of gravity [see for comparison (2.8)], the correlation length $\xi$, or equivalently its inverse $m \equiv 1 / \xi$, is known to be a RG invariant, i.e., $\Lambda \frac{d}{d \Lambda} m(\Lambda, g(\Lambda))=0$. It is the combination of these effects that then leads to an entirely new physical quantum vacuum.

\footnotetext{
${ }^{2}$ The universal quantity $\xi$ also appears in a number of other physical observables, including the exponential decay of the Euclidean correlation function of two infinitesimal loops separated by a distance $|x|$,

$$
G_{\text {loop-loop }}(x)=\left\langle\operatorname{tr} \mathscr{P} \exp \left\{i g \oint_{C_{\varepsilon}} A_{\mu}\left(x^{\prime}\right) d x^{\prime \mu}\right\}(x) \operatorname{tr} \mathscr{P} \exp \left\{i g \oint_{C_{\varepsilon}} A_{\mu}\left(x^{\prime \prime}\right) d x^{\prime \prime \mu}\right\}(0)\right\rangle_{c} .
$$

Here the $C_{\varepsilon}$ 's are two infinitesimal loops centered around $x$ and 0 respectively, suitably defined on the lattice as elementary square loops, and for which one has at sufficiently large separations

$$
G_{\text {loop-loop }}(x) \underset{|x| \rightarrow \infty}{\sim} \exp (-|x| / \xi) .
$$




\section{Gravitational Wilson Loop}

Since the bare cosmological constant can be entirely scaled out of the theory, the legitimate question arises: how can a nonvanishing (and indeed small) effective large-scale cosmological constant arise out of the field-theoretic treatment of quantum gravity? The key to this answer lies in the fact that the lattice field theory itself contains an entirely new dynamically generated scale $\xi$, see Eqs. (2.7) and (2.9).

To see this, consider elementary parallel transports on the lattice. Between any two neighboring pair of simplices $s, s+1$ one can associate a Lorentz transformation $\mathbf{R}_{v}^{\mu}(s, s+1)$. Such a transformation can be directly related to the continuum path-ordered $(\mathscr{P})$ exponential of the integral of the local affine connection $\Gamma_{\mu v}^{\lambda}(x)$ via

$$
R_{v}^{\mu}=\left[\mathscr{P} e^{\int{ }_{\text {between simplices }} \Gamma_{\lambda} d x^{\lambda}}\right]_{v}^{\mu} .
$$

Further one can consider a closed lattice path passing through a large number of simplices $s$, and spanning a large near-planar closed loop $C$. Along $C$ the overall rotation matrix is given by $R_{v}^{\mu}(C)=\left[\prod_{s \subset C} \mathbf{R}_{s, s+1}\right]_{v}^{\mu}$. In a semiclassical picture, if the curvature of the manifold is small, the expression for the full rotation matrix $\mathbf{R}(C)$ associated with the large near-planar loop can be rewritten, using the Stoke's theorem, in terms of a surface integral of the large scale Riemann tensor, projected along the surface area element bivector $A^{\alpha \beta}(C)$ associated with the loop,

$$
R_{v}^{\mu}(C) \approx\left[e^{\frac{1}{2} \int_{S} R^{\cdot} \cdot{ }_{\alpha \beta} A^{\alpha \beta}(C)}\right]_{v}^{\mu} .
$$

The expectation value of the gravitational Wilson loop can be defined in $[11,12,13]$ as $\langle W(C)\rangle=$ $\left\langle\operatorname{tr}\left[B_{C} \mathbf{R}_{1} \mathbf{R}_{2} \ldots \ldots \mathbf{R}_{n}\right]\right\rangle$, where the $\mathbf{R}_{i}$ s are the rotation matrices along the path, and $B_{C}$ related to a constant bivector characterizing the geometric orientation of $C$. One can then show, by using known properties of the Haar measure for the rotation group, that, at least for strong coupling and large area, the gravitational Wilson loop follows an area law, in the same form as in (3.4) with $\xi$ determined, by scaling and dimensional arguments, to be the nonperturbative gravitational correlation length [see (2.7)]. The last result follows from tiling the interior of the given loop by a minimal surface built up of elementary transport loops, in close analogy to the gauge theory case.

One can now compare the quantum result at strong coupling, i.e., the area law, with the semiclassical result that follows from (4.2), which is

$$
W(C) \sim \operatorname{Tr}\left(B_{C} \exp \left\{\frac{1}{2} \int_{S(C)} R_{\cdot \mu \nu} A_{C}^{\mu \nu}\right\}\right) .
$$

Then for a smooth background classical manifold with constant or near-constant large-scale curvature, $R_{\mu v \lambda \sigma}=\frac{1}{3} \lambda\left(g_{\mu v} g_{\lambda \sigma}-g_{\mu \lambda} g_{v \sigma}\right)$, one immediately obtains from the identification of the area terms in the two Wilson loop expressions the following result for the average semiclassical curvature at large scales

$$
\bar{R} \sim+1 / \xi^{2} .
$$

An equivalent way of phrasing the statement of (4.4) uses the classical field equations in the absence of matter, $R=4 \lambda$. The latter suggests one should view $1 / \xi^{2}$ as the observed scaled cosmological 
constant,

$$
\frac{1}{3} \lambda_{o b s} \simeq+\frac{1}{\xi^{2}} .
$$

This last quantity can then be considered as a measure of the gravitational vacuum energy, in analogy to the non-Abelian gauge theory vacuum condensate result, $\left\langle F_{\mu \nu}^{2}\right\rangle \simeq 1 / \xi^{4}$, whose gravity analog can be written, equivalently, as $\langle R\rangle \propto 1 / \xi^{2}$. The nonperturbative treatment of lattice quantum gravity has added one more ingredient to the puzzle: while the bare cosmological constant $\lambda_{0}$ can be completely scaled out of the problem, a new RG invariant scale $\xi$ of (2.7) appears, and is identified with the effective cosmological constant (4.5).

\section{Conclusions}

We examined the role of the cosmological constant in pure Einstein gravity. The key message is that the cosmological constant cannot run with scale, if general covariance is preserved. Instead, evidence from the nonperturbative path integral treatment of quantum gravity points to the fact that the observed effective long-distance cosmological is a renormalization group invariant quantity, related to the fundamental RG scale $\xi$, and thus to a vacuum condensate of the gravitational field. In analogy to the corresponding scale for non-Abelian gauge theories, $\xi$ cannot run, and represents a dynamically generated, nonperturbative mass-like parameter. That this is possible is a highly nontrivial result of the renormalization group treatment, of the Callan-Symanzik RG equations for $G$, and of the phase structure of four dimensional gravity.

\section{References}

[1] S. Weinberg, Ultraviolet divergences in quantum gravity, in 'General Relativity - An Einstein Centenary Survey', edited by S. W. Hawking and W. Israel,(Cambridge University Press, 1979).

[2] R. Gastmans, R. Kallosh and C. Truffin, Nucl. Phys. B 133417 (1978).

[3] S. M. Christensen and M. J. Duff, Phys. Lett. B 79213 (1978).

[4] R. Gastmans, R. Kallosh and C. Truffin, Nucl. Phys. B 133417 (1978).

[5] S. M. Christensen and M. J. Duff, Phys. Lett. B 79213 (1978).

[6] H. Kawai and M. Ninomiya, Nucl. Phys. B 336, 115 (1990).

[7] H. W. Hamber and R. M. Williams, Nucl. Phys. B 248, 392 (1984).

[8] H. W. Hamber and R. M. Williams, Phys. Lett. B 157, 368 (1985).

[9] H. W. Hamber and R. M. Williams, Nucl. Phys. B 269, 712 (1986).

[10] K. G. Wilson, Phys. Rev. D 10, 2445 (1974).

[11] H. W. Hamber and R. M. Williams, Phys. Rev. D 73, 044031 (2006).

[12] H. W. Hamber and R. M. Williams, Phys. Rev. D 76, 084008 (2007).

[13] H. W. Hamber and R. M. Williams, Phys. Rev. D 81, 084048 (2010).

[14] H. W. Hamber, Quantum Gravitation, Springer Tracts in Modern Physics, ISBN-10: 3540852921 (Springer, New York, 2009).

[15] A. O. Barvinsky, Phys. Lett. B 572109 (2003). 